ISSN 1810-3030 (Print) 2408-8684 (Online)

Journal of Bangladesh Agricultural University

Journal home page: http://baures.bau.edu.bd/jbau, www.banglajol.info/index.php/JBAU

\title{
Weed diversity of the family Poaceae in Bangladesh Agricultural University campus and their ethnobotanical uses
}

\section{${ }^{\otimes}$ Ashaduzzaman Sagar, Jannat-E-Tajkia and A.K.M. Golam Sarwar}

Laboratory of Plant Systematics, Department of Crop Botany, Bangladesh Agricultural University, Mymensingh

\begin{tabular}{|c|c|}
\hline ARTICLE INFO ofen ${ }_{\text {access }}$ & Abstract \\
\hline $\begin{array}{l}\text { Article history: } \\
\text { Received: 03 July } 2018 \\
\text { Accepted: } 19 \text { November } 2018 \\
\text { Published: } 31 \text { December } 2018 \\
\end{array}$ & $\begin{array}{l}\text { A taxonomic study on the weeds of the family Poaceae growing throughout the Bangladesh Agricultural } \\
\text { University campus was carried out to determine species diversity of grasses in the campus. A total of } 81 \\
\text { species under } 46 \text { genera and } 2 \text { subfamilies of the family Poaceae were collected and identified; their uses } \\
\text { in various ailments were also recorded. Out of the three subfamilies, no weed from the subfamily } \\
\text { Bambusoideae was found. Among the genera, Digitaria, Eragrostis, Brachiaria, Panicum, Echinochloa } \\
\text { and Sporobolus were most dominant in context to number of snecies with a total of } 29 \text { snecies. While } 28\end{array}$ \\
\hline $\begin{array}{l}\text { Keywords: } \\
\text { Grass weeds; Phenology; } \\
\text { Taxonomy; BAU campus; } \\
\text { Ethnobotanical uses }\end{array}$ & $\begin{array}{l}\text { genera were represented by single species each in BAU campus; of these } 15 \text { genera were in Bangladesh as } \\
\text { well. Some of them are major and obnoxious weeds in different crop fields including staples rice and } \\
\text { wheat. The flowering period will be helpful for the management of respective weed population. Many of } \\
\text { these weed species have high economical, ethnomedicinal and other uses. The phenological study of these }\end{array}$ \\
\hline Corre & intry without affecting the agro-ecosystem by keeping the weed population below a threshold level. \\
\hline
\end{tabular}

凶: sagar@bau.edu.bd

\section{Copyright: \\ (c) (i)}

(C) 2018 by authors and BAURES. This work is licensed under the Creative Commons Attribution International License (CC By 4.0).

\section{Introduction}

The family Poaceae (conserved name Gramineae), universally known as grass family, is the fifth largest plant family, following the Asteraceae, Orchidaceae, Fabaceae and Rubiaceae. It is the second most diverse family among monocotyledons (Bouchenak-khelladi et $a l ., 2010)$, with 780 genera and around 12,000 species of cosmopolitan distribution (Christenhusz and Byng, 2016). They are most plentiful in tropical and northtemperate semi-arid regions with seasonal rainfall. In Bangladesh, the family is represented by 3 subfamilies viz. Bambusoideae, Panicoideae and Pooideae, with 113 genera and 285 species (Ahmed et al., 2008). All staple food and cereals like rice, wheat, corn, etc. is from this family. These grasses provide a vegetative cover, feed/fodder for animals, controls nutrient cycling, adds organic matter, acts as a soil binder, serves as an important source of genetic materials for crop improvement and used in the beautification of the landscapes. On the other hand, about $25 \%$ weeds of the world are monocotyledonous (Bryson and Carter, 2008); and grasses are one of the most troublesome and difficult group to control (Lym and Travnicek, 2015) because of their highly adaptive mechanisms and allelopathic properties (Noor et al., 2012). They sometimes act as an alternative host of different diseases and pests in the absence of main crops. The outbreak of diseases occurs again when the relevant agricultural crops are cultivated in the field. Thereby, the cost of production is increased and the quality of the products is reduced.

Bangladesh Agricultural University (BAU) is the largest university in Bangladesh and it covers about 486 hectares of land. It is situated in the Mymensingh division under the Old Brahmaputra Floodplain, Agroecological zone 9, of Bangladesh (UNDP, 1988). Three major topographic types like plain area, slightly undulated area and basin-shaped low lying area are present in the BAU campus which facilitates a wide range of habitats such as wetlands, marshy lands, open fields, bare lands, etc. (Sarwar and Prodhan, 2011; Jannat-E-Tajkia et al., 2018). The soil category of the region varies from clay, clay loam to sandy loam (UNDP, 1988). The mean annual rainfall is $244.15 \mathrm{~mm}$ and the temperature varies from $11.9^{\circ}$ to $32.5^{\circ} \mathrm{C}$. The weather of this campus is not characterized by large extremes of heat, cold and rainfall. The winter period (November-December) is cool and almost rainless; whereas the summer season (June-October) is hot and humid, and during summer $90 \%$ or more of the whole rain occurs. This diverse climatic condition provides a suitable condition for the growth of grasses in the BAU campus.

In Bangladesh, the detailed taxonomic studies of weeds have been neglected by both the practising plant taxonomists and/or the agricultural scientists. Despite 
the plenty of grasses in BAU campus, no momentous taxonomic study on grasses of this region has been made in the current years but only weeds of different specific crops were studied (Shabi et al., 2018). Some studies were carried out on the occurrences of sedges in the BAU campus (Sarwar and Prodhan, 2011; Jannat-ETajkia et al., 2018), but the species diversity of grasses in the campus was not conducted. Therefore, the current survey aimed at making an intensive taxonomic study of the weeds of Poaceae family in the BAU campus including their habitat, flowering period, ethnobotanical uses and economic importance; which may be useful for the green management practices and for getting higher economic benefits.

\section{Materials and Methods}

A rigorous field survey was carried out of the weeds of Poaceae family grown in BAU campus during 2015 to 2016. During the survey, fresh flowering samples were collected through the year round by frequent field visits (once a week). Other related information e.g., habitat, location, date, flowering time, crop/plant association, etc. were recorded during the field collection and by searching from published literature and online resources. Fresh samples were dried well for making voucher specimens. The collected fresh or dried specimens were identified by matching with herbarium specimens or published literature or consulting with experienced taxonomist at the Bangladesh National Herbarium, Dhaka. All the specimens are preserved in Prof. Dr. Arshad Ali Herbarium at the Botanical Garden, Department of Crop Botany, Bangladesh Agricultural University. The information related to use were collected from the published literature (Ahmed et al., 2008; Sarwar and Prodhan, 2011).

\section{Results and Discussion}

A sum of 81 grass weed species under 46 genera and 2 sub-families of the family Poaceae were found in BAU campus (Table 1). The subfamily Panicoideae was represented by 31 genera and the subfamily Pooideae by 15 genera. Though crops from the subfamily Bambusoideae were present in the campus, weeds from this group were not found (Fig. 1) due to the limited selection of crops for the cultivation. Among the 46 genera, the most dominant 6 genera were Digitaria with 9 species, followed by Brachiaria (5), Panicum (3), and Echinochloa (3) which are from the subfamily Panicoideae and Eragrostis (6) and Sporobolus (3) are from the subfamily Pooideae. Moreover, these six genera together account for 29 species (35.8\%) out of 81 weed species of this family in the BAU campus (Table 1). Again 28 genera were represented by single species each in BAU campus; of which 15 genera are represented by single species in Bangladesh as well. They are Axonopus, Dimera, Erianthus, Heteropogon, Ichnanthus, Imperata, Ottochloa, Pseudechinolaena,
Urochloa, Zoysia, Aeluropus, Dactyloctenium, Elytrophorus, Hygroryza and Leersia (Ahmed et al., 2008; Table 1). The first nine of them were from Panicoideae and the rest were from the subfamily Pooideae.

The findings of this study revealed that some of these weeds are very common and major weeds in the rice, wheat, jute and other crop fields (Table 1; Bor, 1960; Gilliland, 1971). The most important grass weeds of Poaceae family in terms of their adverse effect on agriculture include Cynodon dactylon, Echinochloa crus-galli, E. colona, Eleusine indica, Imperata cylindrica, ranking $2^{\text {nd }}, 3^{\text {rd }}, 4^{\text {th }}, 5^{\text {th }}$, and $7^{\text {th }}$ among the world's worst weeds, respectively (Holm et al., 1977; USDA, 2012). Some of them are very common weeds in garden crops like Digitaria sanguinalis, Pennisetum glaucum, etc. All of them are present in BAU campus (Table 1).

The ethnomedicinal and other uses of weeds from Poaceae family are much diversified (Table 1; Fig. 2) and also recorded in different ethnobotanical references (Uddin, 2006; Ahmed et. al., 2008). Some of them are very palatable fodder for cows, buffaloes, elephants, etc. Many of them are good soil binder and used as raw materials for paper industry, to decorate and soil binder for lawn and turf (Table 1). They have also a great medicinal use. Some of them are tonic for bone fracture, rat bite, bellyache after child birth, internal hemorrhage, convulsion, constipation, cardiac and liver diseases, etc. (Table 1; Fig. 3).

The proper management activities for weedy grasses are diverse. Cultural methods such as roguing, hoeing, tillage, different mulches or cover crop, draft ploughing, etc. are still used in the world to manage weeds including grasses and sedges (Shear, 1985). But, a cultural method of control such as mowing alone will not successfully manage certain perennial grass weeds rather it can prevent seed production if mowing intervals are shorter than the time necessary to set fertile spikelets (Shear, 1985). Therefore, it is necessary to know flowering periods to control the grass weeds. The phenologies were recorded here could be used to control them and these showed a wide variation (Table 1). Therefore, the grass weed populations can be managed below the critical level if proper steps can be taken before flowering. Therefore, the judicious and improved cropping pattern should be developed for additional high economic return from grass weeds of the family Poaceae without hampering our agro-ecosystem and crop yield as well. 


\section{Table 1. Weeds from the family Poaceae recorded in the Bangladesh Agricultural University campus}

\begin{tabular}{|c|c|c|c|c|c|c|}
\hline Sl. & Botanical Name & Bengali Name & English Name & Flowering Period & Habitat & Ethnobotanical \& Economic Importance \\
\hline 1 & $\begin{array}{l}\text { Acroceras munroanum (Balansa) } \\
\text { Henrard }\end{array}$ & - & - & April-August & $\begin{array}{l}\text { Low, moist fields, margin of ponds, } \\
\text { rice fieldsetc. }\end{array}$ & Fodder (Gilliland, 1971) \\
\hline 2 & Acroceras zizanioides (Kunth) Dandy & - & Oat grass & June-October & $\begin{array}{l}\text { Roadside, in or around watermarshy } \\
\text { land, shady area etc. }\end{array}$ & Fodder (Gilliland, 1971) \\
\hline 3 & Aeluropus lagopoides (L.) Thwaites & Nona durba & Mangrove grass & April-October & $\begin{array}{l}\text { Low-lying places, sandy soils and arid } \\
\text { places }\end{array}$ & $\begin{array}{l}\text { Good fodder (Gilliland, 1971), Soil binder (Bor, } \\
\text { 1960) }\end{array}$ \\
\hline 4 & Arthraxon lancifolius (Trin.) Hochst. & - & - & August-December & $\begin{array}{l}\text { Moist \& shady situations, high land } \\
\text { slopes, old fences }\end{array}$ & Fodder (Bor, 1960), Healing for disease \\
\hline 5 & Arthraxon nudus (Steud.) Hochst. & Gandha Bena & - & September-March & $\begin{array}{l}\text { Moist \& shady situations, high land } \\
\text { slopes, old fences }\end{array}$ & Soil binder (Bor, 1960) \\
\hline 6 & $\begin{array}{l}\text { Arundinella bengalensis (Spreng.) } \\
\text { Druce }\end{array}$ & Ganga Bena & - & October-February & Railway side, high-land areas & Weed \\
\hline 7 & Axonopus compressus (Sw.) P. Beauv. & Ghora-dubo Har & $\begin{array}{l}\text { Carpet/Blanket/Savann } \\
\text { ah grass }\end{array}$ & Year around & Wide range of habitat and soils & $\begin{array}{l}\text { Weed, Fodder (Gilliland, 1971), used in bone crack } \\
\text { (Uddin, 2006). }\end{array}$ \\
\hline 8 & Bothriochloa pertusa (L.) A. Camus & Barboda ghas & $\begin{array}{l}\text { Hurricane/Barbados- } \\
\text { sour grass }\end{array}$ & August-February & $\begin{array}{l}\text { Bunds of fields, wetlands and open } \\
\text { grassland }\end{array}$ & Fodder, Hay, Pasture (Bor, 1960) \\
\hline 9 & Brachiaria brizantha (A. Rich.) Stapf & - & $\begin{array}{l}\text { Bread Grass, Palisade } \\
\text { Grass }\end{array}$ & September-December & $\begin{array}{l}\text { Open and moist places with medium } \\
\text { to high rainfall }\end{array}$ & $\begin{array}{l}\text { Fodder, Hay, Soil binder (Bor, 1960; Skerman and } \\
\text { Riveros, 1990) }\end{array}$ \\
\hline 10 & Brachiaria distachya (L.) Stapf & Corighas & Cori grass & November-March & Bank of waterlands \& wastelands & Fodder, Soil binder (Bor, 1960) \\
\hline 11 & Brachiaria mutica (Frossk.) Stapf & $\begin{array}{l}\text { Nardul, Para } \\
\text { ghas }\end{array}$ & $\begin{array}{l}\text { Para grass, Buffalo } \\
\text { grass }\end{array}$ & November-March & $\begin{array}{l}\text { Moist and wet grounds, bank of rivers, } \\
\text { canals etc. }\end{array}$ & Weed, Fodder, Soil binder (Bor, 1960) \\
\hline 12 & $\begin{array}{l}\text { Brachiaria reptans } \\
\text { C.E.Hubb. }\end{array}$ & Para ghas & $\begin{array}{l}\text { Creeping panic or } \\
\text { running grass }\end{array}$ & August-September & $\begin{array}{l}\text { Water-logged areas, wetlands \& shade } \\
\text { of bushes }\end{array}$ & Weed, Fodder (Bor, 1960) \\
\hline 13 & $\begin{array}{l}\text { Brachiaria subquadripara (Trin.) } \\
\text { Hitchc. }\end{array}$ & Cori ghas & Green-summer grass & November-March & $\begin{array}{l}\text { Bank of waterlands, wastelands \& } \\
\text { coastal areas }\end{array}$ & Fodder, Soil binder (Bor, 1960) \\
\hline 14 & Cenchrus ciliaris $\mathrm{L}$. & Anjan Ghas & African Foxtail & Year round & $\begin{array}{l}\text { Water-logged areas, wetlands \& shade } \\
\text { of bushes }\end{array}$ & Excellent Fodder, Hay, Lawn grass (Bor, 1960) \\
\hline 15 & Centotheca lappacea (L.) Desv. & - & Barbed grass & October-March & $\begin{array}{l}\text { Wet zone, mostly in moist \& shady } \\
\text { floor }\end{array}$ & Excellent Fodder (Bor, 1960) \\
\hline 16 & Chloris barbata $\mathrm{Sw}$. & - & Swollen finger grass & April-May & $\begin{array}{l}\text { Roadsides, open field, crop field and } \\
\text { wetlands }\end{array}$ & Fodder at young (Bor, 1960) \\
\hline 17 & Chloris gayana Kunth & - & Rhode's Grass & November-February & $\begin{array}{l}\text { Grassland, open woodland, marshy } \\
\text { land }\end{array}$ & $\begin{array}{l}\text { Hay, Pasture lye, Soil binder, OM (Skerman and } \\
\text { Riveros, 1990) }\end{array}$ \\
\hline 18 & Chrysopogon aciculatus (Retz.) Trin. & $\begin{array}{l}\text { Premkanta, } \\
\text { Chorkanta }\end{array}$ & Grass Seed, Love Grass & Year around & Roadsides \& railway sides & $\begin{array}{l}\text { Weed, lawn grass (Bor, 1960), used in rat bite } \\
\text { (Uddin, 2006) }\end{array}$ \\
\hline 19 & Cynodon dactylon (L.) Pers & $\begin{array}{l}\text { Durba/Dubba } \\
\text { ghas }\end{array}$ & $\begin{array}{l}\text { Bahama grass, } \\
\text { Bermuda grass }\end{array}$ & July-December & $\begin{array}{l}\text { Cultivated lands, moist or dry waste } \\
\text { places, roadsides, lawn, and riversides }\end{array}$ & $\begin{array}{l}\text { Weed, curing bellyache after childbirth (Kirtikar et } \\
\text { al., 1935), Fish poisoning and ureterolithiasis (Pal } \\
\text { and Jain, 1998). Stop bleeding, Lawn grass, Soil } \\
\text { binder (Bor, 1960). }\end{array}$ \\
\hline 20 & Cynodon radiatus Roth & Nil durba & Bahama grass & March-September & Banks of rivers and rarely roadsides & Weed \\
\hline 21 & Cyrtococcum oxyphyllum (Steud.) Stapf & Oxycocca Ghash & - & Year around & $\begin{array}{l}\text { Marshy land and occasionally in } \\
\text { grasslands }\end{array}$ & Good fodder (Gilliland, 1971) \\
\hline 22 & Cyrtococcum patens (L.) A. Camus & Patcocca ghas & - & Year around & $\begin{array}{l}\text { Shady habitat, especially as } \\
\text { undergrowth in tree plant }\end{array}$ & Excellent fodder (Gilliland, 1971) \\
\hline 23 & Dactyloctenium aegyptium (L.) Willd & Makra & $\begin{array}{l}\text { Crowfoot grass, Beach } \\
\text { wire-grass, Giant } \\
\text { Button-grass }\end{array}$ & Year around & Sandy places of the lowlands & $\begin{array}{l}\text { Lawn grass, fodder (Bor, 1960), Curing bellyache } \\
\text { after childbirth (Kirtikar et al., 1935), Fish } \\
\text { poisoning and ureterolithiasis (Pal and Jain, 1998). }\end{array}$ \\
\hline
\end{tabular}


Table 1. Contd.

\begin{tabular}{|c|c|c|c|c|c|c|}
\hline SI. & Botanical Name & Bengali Name & English Name & Flowering Period & Habitat & Ethnobotanical \& Economic Importance \\
\hline 24 & Dichanthium annulatum (Forssk) Stapf & Loari & $\begin{array}{l}\text { Sheda grass, Kleberg } \\
\text { stem }\end{array}$ & January-June & Limestone hills and open grasslands & Excellent fodder, Erosion control (Bor, 1960). \\
\hline 25 & Dichanthium caricosum (L.) A. Camus & Detara & $\begin{array}{l}\text { Antigua Hay-grass, } \\
\text { Blue grass }\end{array}$ & November-January & Open sunny places in dry season & $\begin{array}{l}\text { Palatable fodder, Soil binder (Skerman and } \\
\text { Riveros, 1990) }\end{array}$ \\
\hline 26 & $\begin{array}{l}\text { Digitaria abludens (Roem. \& Schult.) } \\
\text { Veldkamp }\end{array}$ & Chirichira & - & July-August & $\begin{array}{l}\text { Open spaces, and fields of variety of } \\
\text { soils }\end{array}$ & Weed \\
\hline 27 & $\begin{array}{l}\text { Digitaria bicornis (Lam.) Roem. \& } \\
\text { Schult. }\end{array}$ & - & Asian crab grass & March-September & $\begin{array}{l}\text { Crop fields, roadsides, gardens } \\
\text { andpastures }\end{array}$ & Fodder at young but weed at mature (Bor, 1960) \\
\hline 28 & Digitaria ciliaris (Retz). Koeler & $\begin{array}{l}\text { Makur-jali, } \\
\text { Kokjachira }\end{array}$ & Hairy/Wild Crab Grass & Year around & $\begin{array}{l}\text { Wastelands, riverbanks, moist shady } \\
\text { places and roadsides }\end{array}$ & Weed, Fodder grass (Skerman and Riveros, 1990) \\
\hline 29 & Digitaria ischaemum (Schreb.) Muhl. & - & $\begin{array}{l}\text { Smooth/small Crab } \\
\text { Grass }\end{array}$ & June-August & $\begin{array}{l}\text { Open spaces, and fields of variety of } \\
\text { soils }\end{array}$ & Weed \\
\hline 30 & Digitaria longiflora (Retz.) Pers. & - & $\begin{array}{l}\text { False couch finger } \\
\text { grass/ Indian crab grass }\end{array}$ & Year around & Roadsides and open grounds & Common fodder (Bor, 1960) \\
\hline 31 & Digitaria radicosa (J.Presl) Miq. & $\begin{array}{l}\text { Timorese crab } \\
\text { grass }\end{array}$ & Trailing crabgrass & $\begin{array}{l}\text { September- } \\
\text { November }\end{array}$ & $\begin{array}{l}\text { Shady places, river banks \& } \\
\text { wastelands }\end{array}$ & Weed, Fodder (Bor, 1960) \\
\hline 32 & Digitaria sanguinalis (L.) Scop. & Makunjali & $\begin{array}{l}\text { Crab grass, Finger- } \\
\text { grass }\end{array}$ & August-October & $\begin{array}{l}\text { Cultivated and waste places, railway } \\
\text { sides \& dumps }\end{array}$ & Important fodder (Bor, 1960) \\
\hline 33 & Digitaria setigera Roth & Sheti ghas & East Indian crab grass & Year around & $\begin{array}{l}\text { River banks, wastelands, roadsides, } \\
\text { crop fields etc. }\end{array}$ & Weed \\
\hline 34 & Digitaria stricta Roth & Trick ghas & - & September-December & Lawns or cultivated lands & Good fodder grass (Bor, 1961) \\
\hline 35 & Dimera ornithopoda Trin. & Pakhi daimara & - & August-March & $\begin{array}{l}\text { Dry grassy spots of lowland, } \\
\text { roadsides, broken wall }\end{array}$ & Weed \\
\hline 36 & Echinochloa colona (L.) Link & $\begin{array}{l}\text { Khudey shama } \\
\text { ghas, shama } \\
\text { dhan }\end{array}$ & $\begin{array}{l}\text { Awnless barnyard } \\
\text { grass, Jungle rice }\end{array}$ & May- September & $\begin{array}{l}\text { Paddy fields, wastelands, fallow lands } \\
\text { and shallow marshes }\end{array}$ & $\begin{array}{l}\text { Weed of rice, fodder, vegetable (Pal and Jain, } \\
\text { 1998). Helps in digestion }\end{array}$ \\
\hline 37 & Echinochloa crus-galli (L.) P.Beauv. & $\begin{array}{l}\text { Bara shama } \\
\text { ghas }\end{array}$ & $\begin{array}{l}\text { Barnyard-millet, } \\
\text { Cockspur grass, water } \\
\text { grass }\end{array}$ & April-October & $\begin{array}{l}\text { Marshy and muddy waste lands and } \\
\text { rice fields }\end{array}$ & $\begin{array}{l}\text { Worst weed of paddy (Skerman and Riveros, 1990) } \\
\text { and Vegetables (Purseglove, 1968). Grain fodder } \\
\text { (Bor, 1960), and used in internal haemorrhage } \\
\text { (Kirtikar et al., 1935). }\end{array}$ \\
\hline 38 & Echinochloa stagnina (Retz.) P.Beauv. & Dul, Parua & $\begin{array}{l}\text { Floating barnyard/ } \\
\text { hippo/ burgu grass }\end{array}$ & March-September & $\begin{array}{l}\text { Marshy places, wetlands \&paddy } \\
\text { fields }\end{array}$ & $\begin{array}{l}\text { Weed of paddy, fodder with high sugar (Bor, 1960; } \\
\text { Purseglove, 1968) }\end{array}$ \\
\hline 39 & Eleusine indica (L.) Gaertn. & $\begin{array}{l}\text { Ghora } \\
\text { dubboher, Mal- } \\
\text { ankuri. }\end{array}$ & $\begin{array}{l}\text { Crab grass, Goose } \\
\text { grass, Crowfoot grass }\end{array}$ & June-August & $\begin{array}{l}\text { Dry and wetlands, lawns, vegetable } \\
\text { gardens, roadsides, wastelands and } \\
\text { lowlands }\end{array}$ & $\begin{array}{l}\text { Weed, Fodder with cyanogenetic effect but used at } \\
\text { young (Bor, 1960). Used in convulsion, liver } \\
\text { disorder \& bone fracture (Kirtikar et al., 1935) }\end{array}$ \\
\hline 40 & Elytrophorus spicatus (Willd.) A.Camus & Jangli rala & Spike grass & December-February & $\begin{array}{l}\text { Low flooded clayey sites, lowlands } \\
\text { and mid-uplands }\end{array}$ & Weed \\
\hline 41 & Eragrostis cilianensis (All.) Janch. & - & $\begin{array}{l}\text { Stink grass, Grey love } \\
\text { grass }\end{array}$ & June-November & Undergrowth of trees and high land & Occasionally fodder (Bor, 1960) \\
\hline 42 & Eragrostis coarctata Stapf & Jinkua & Love grass & November-February & Dry places & Weed \\
\hline 43 & Eragrostis gangetica (Roxb.) Steud. & Jinkua, Khari & Slim flower love grass & June-December & $\begin{array}{l}\text { Lowlands, damp places, river beds and } \\
\text { paddy fields }\end{array}$ & Weed \\
\hline 44 & Eragrostis japonica (Thunb.) Trin. & - & Pond lovegrass & September-January & $\begin{array}{l}\text { Damp soil near water-logged places, } \\
\text { rivers and broken wall }\end{array}$ & Occasionally fodder (Bor, 1960) \\
\hline 45 & Eragrostis pilosa (L.) P.Beauv. & - & Indian/Soft lovegrass & May-August & $\begin{array}{l}\text { Sides of rivers, ponds, dry places and } \\
\text { roadside. }\end{array}$ & Weed \\
\hline 46 & $\begin{array}{l}\text { Eragrostis uniloides (Retz.) Nees } e x \\
\text { Steud. }\end{array}$ & Koni & Chinese lovegrass & Year around & $\begin{array}{l}\text { Roadsides, banks of rivers and } \\
\text { cultivated fields }\end{array}$ & $\begin{array}{l}\text { Cosmopolitan weed, Fodder, Green manure } \\
\text { (Mannetje and Jones, 1992) }\end{array}$ \\
\hline
\end{tabular}




\section{Table 1. Contd.}

\begin{tabular}{|c|c|c|c|c|c|c|}
\hline Sl. & Botanical Name & Bengali Name & English Name & Flowering Period & Habitat & Ethnobotanical \& Economic Importance \\
\hline 47 & Erianthus longisetus Andersson & - & - & November-April & High land area & Weed \\
\hline 48 & $\begin{array}{l}\text { Eriochloa fatmensis (Hochst. \& Steud.) } \\
\text { Clayton }\end{array}$ & - & - & May-August & Wet and dry soil & Fodder of stock (Skerman and Riveros, 1990) \\
\hline 49 & Eriochloa procera (Retz.) C.E.Hubb. & Pelu, Kap ghas & Cup grass & April-October & $\begin{array}{l}\text { Damp places, paddy fields and banks } \\
\text { of backwater. }\end{array}$ & Quick growing and succulent fodder \\
\hline 50 & Eulalia conorta (Brongn.) Kuntze & Eulali Ghash & Golden velvet grass & September-February & Open grassy area and marshy land & Weed \\
\hline 51 & Eulalia leschenaultiana (Decne.) Ohwi & - & - & November-January & Bunds of rice fields and roadsides & Weed \\
\hline 52 & Hemarthria protensa Steud. & Chailla & Joint grass & May-October & Marshes, plain and high land & Weed \\
\hline 53 & $\begin{array}{l}\text { Heteropogon contortus (L.) P. Beauv. } \\
\text { ex Roem. \& Schult. }\end{array}$ & Shukla, Kusal & $\begin{array}{l}\text { Pili grass, Black spear } \\
\text { grass }\end{array}$ & August-January & $\begin{array}{l}\text { Undergrowth of tree plants, marshy } \\
\text { land }\end{array}$ & $\begin{array}{l}\text { Seed wool fatal to sheep, can cause skin and eye } \\
\text { disorder (Tothill and Backer, 1983) }\end{array}$ \\
\hline 54 & $\begin{array}{l}\text { Hygroryza aristata (Retz.) Nees } e x \\
\text { Wight \& Arn. }\end{array}$ & $\begin{array}{l}\text { Jangli dhan, } \\
\text { Phutki }\end{array}$ & Bengal wild rice & October-February & $\begin{array}{l}\text { Dry area, tanks and marshes, paddy } \\
\text { fields }\end{array}$ & $\begin{array}{l}\text { Fodder (Bor, 1960), causes flatulence \& } \\
\text { constipation (Kirtikar } \text { et al., 1935) }\end{array}$ \\
\hline 55 & $\begin{array}{l}\text { Ichnanthus pallens (Sw.) Munro ex } \\
\text { Benth. }\end{array}$ & - & - & March-August & $\begin{array}{l}\text { Damp, shady sites, mainly in wet } \\
\text { region }\end{array}$ & Highly palatable fodder (Bor, 1960) \\
\hline 56 & Imperata cylindrica (L.) Raeusch. & $\begin{array}{l}\text { Chhan, Chau, } \\
\text { Kash, Sarkanb }\end{array}$ & $\begin{array}{l}\text { Cogon grass, Cotton } \\
\text { wool-grass, Blady } \\
\text { grass, Spear grass }\end{array}$ & Year around & Open tree area and roadside areas & $\begin{array}{l}\text { Noxious weed in rice, tea, coffee, coconut, rubber, } \\
\text { teak etc. Thaching grass, Paper industry, Fodder } \\
\text { (Bor, 1960), Soil binder, Bouquet material, used in } \\
\text { curing chest cold (Skerman and Riveros, 1990). }\end{array}$ \\
\hline 57 & Isachne globosa (Thunb.) Kuntze & - & Swamp millet & Year around & $\begin{array}{l}\text { Marshy places, rice fields, bank of } \\
\text { riversand ditches }\end{array}$ & $\begin{array}{l}\text { Weed of paddy, Fodder and Green manure } \\
\text { (Skerman and Riveros, 1990). }\end{array}$ \\
\hline 58 & Isachne scabrosa Hook. f. & - & - & September-December & Open grassland and high land regions & Weed \\
\hline 59 & Leersia hexandra Sw. & $\begin{array}{l}\text { Arali ghas, } \\
\text { Jungli dhan }\end{array}$ & $\begin{array}{l}\text { Lambedora grass, } \\
\text { Swamp rice grass }\end{array}$ & August-June & $\begin{array}{l}\text { Sides of streams, backwater, canals, } \\
\text { fallow lands }\end{array}$ & $\begin{array}{l}\text { Weed of paddy, Fodder, Food of rodents (Skerman } \\
\text { and Riveros, 1990) }\end{array}$ \\
\hline 60 & Leptochloa panicea (Retz.) Ohwi & - & Mucronate Sprangletop & May-October & Dry waste places & Weed \\
\hline 61 & Oplismenus burmanni (Retz.) P.Beauv. & Kombo zara & $\begin{array}{l}\text { Wavy-leaf/ Burmann's } \\
\text { basketgrass }\end{array}$ & September-January & $\begin{array}{l}\text { Bunds, roadside, high land area, moist, } \\
\text { shady places etc. }\end{array}$ & Fodder (Bor, 1960) \\
\hline 62 & Oplismenus compositus (L.) P.Beauv & Bashawa, Gohur & Bamboo leaf grass & August-September & $\begin{array}{l}\text { Undergrowth in tree plants and other } \\
\text { shady places }\end{array}$ & Weed \\
\hline 63 & Ottochloa nodosa (Kunth) Dandy & - & Slender panic grass & July-December & $\begin{array}{l}\text { Lowlands, muddy river banks, wet } \\
\text { sites etc. }\end{array}$ & Eaten by stock (Bor, 1960) \\
\hline 64 & Panicum brevifolium $\mathrm{L}$. & - & Shortleaf panic grass & March-December & $\begin{array}{l}\text { Shady places of trees, riverbanks and } \\
2^{\circ} \text { vegetation }\end{array}$ & Fodder (Bor, 1960) \\
\hline 65 & Panicum paludosum Roxb. & Barti, Borali & $\begin{array}{l}\text { Marsh/Swamp panic } \\
\text { grass }\end{array}$ & Year around & $\begin{array}{l}\text { Wet lands, marshes, and banks of } \\
\text { backwater }\end{array}$ & $\begin{array}{l}\text { Good Fodder for buffaloes and elephants (Bor, } \\
\text { 1960) }\end{array}$ \\
\hline 66 & Panicum repens $\mathrm{L}$. & $\begin{array}{l}\text { Baranda, Dhani } \\
\text { ghas }\end{array}$ & $\begin{array}{l}\text { Creeping panicum } \\
\text { grass }\end{array}$ & Year around & $\begin{array}{l}\text { Roadsides, margin of ditches, tanks, } \\
\text { marshes, rice fields }\end{array}$ & $\begin{array}{l}\text { Nutritious pasture (Bor, 1960), Used in eye disease } \\
\text { (Pal and Jain, 1998). }\end{array}$ \\
\hline 67 & Paspalum conjugatum P.J. Bergius & - & $\begin{array}{l}\text { Buffalo grass, Carabao } \\
\text { grass }\end{array}$ & Year around & $\begin{array}{l}\text { Plantations, lawns, roadsides, other } \\
\text { open grassy areas etc. }\end{array}$ & Weed of rice, Fodder and Lawn grass \\
\hline 68 & $\underline{\text { Pennisetum glaucum (L.) R.Br. }}$ & $\begin{array}{l}\text { Bajra, Kauni, } \\
\text { Banaspati ghas }\end{array}$ & $\begin{array}{l}\text { Bulrush millet, Pearl } \\
\text { millet, Cumboo millet }\end{array}$ & September-December & Plains and hills & $\begin{array}{l}\text { Forage (Bor, 1961), stalk as bedding, fencing and } \\
\text { fuel (Skerman and Riveros, 1990), tonic in cardiac } \\
\text { (Kirtikar et al., 1935) and sexual disease (Pal and } \\
\text { Jain, 1968) }\end{array}$ \\
\hline 69 & Pogonatherum crinitum (Thunb.) Kunth & Sunali-gash & Bamboo grass & Year around & $\begin{array}{l}\text { Tracks and banks of river and streams, } \\
\text { broken wall, crevices of rocks and in } \\
\text { shady places. }\end{array}$ & Weed \\
\hline 70 & Pogonatherum paniceum (Lam) Hack. & - & $\begin{array}{l}\text { Dwarf bamboo, Golden } \\
\text { hair grass }\end{array}$ & March-September & Dry high land & Ornamental grass (Skerman and Riveros, 1990) \\
\hline
\end{tabular}




\section{Table 1. Contd.}

\begin{tabular}{|c|c|c|c|c|c|c|}
\hline Sl. & Botanical Name & Bengali Name & English Name & Flowering Period & Habitat & Ethnobotanical \& Economic Importance \\
\hline 71 & $\frac{\text { Pseudechinolaena polystachya (Humb., }}{\text { Bonpl. \& Kunth) Stapf }}$ & - & - & Year around & Shades of trees & Weed \\
\hline 72 & $\begin{array}{l}\text { Pseudoraphis spinsescens (R.Br.) } \\
\text { Vickery }\end{array}$ & - & Spiny mudgrasses & May-September & $\begin{array}{l}\text { Stagnant water bodies, slow moving } \\
\text { rivers and muddy surface of dried up } \\
\text { area }\end{array}$ & Weed \\
\hline 73 & Sacciolepis indica (L.) Chase & - & Glenwood grass & May-December & $\begin{array}{l}\text { Moist grassy slopes with partial } \\
\text { shades }\end{array}$ & Give grazing (Gilliland, 1971) \\
\hline 74 & $\begin{array}{l}\text { Sacciolepis myosuroides (R.Br.) A. } \\
\text { Camus }\end{array}$ & Hill tauta & Cupscale grass & August-December & $\begin{array}{l}\text { Moist or wet sites, marshes and rice } \\
\text { fields }\end{array}$ & Weed of cultivated lands \\
\hline 75 & Schizachyrium brevifolium (Sw.) Buse & - & Serillo Dulce & October-December & $\begin{array}{l}\text { Lowlands, cultivated lands and open } \\
\text { sandy area. }\end{array}$ & Poor fodder (Gilliland, 1971) \\
\hline 76 & $\underline{\text { Setaria viridis (L.) P.Beauv. }}$ & Bhagar & Green foxtail & August-December & $\begin{array}{l}\text { Bunds of paddy fields, roadsides and } \\
\text { wastelands. Usually in moist habitat }\end{array}$ & Fodder (Bor, 1961) \\
\hline 77 & Sporobolus diandrus (Retz.) P.Beauv. & Bina joni & Indian dropseed & March-October & Cultivated lands, grasslands, roadsides & Good fodder (Bor, 1961) \\
\hline 78 & Sporobolus indicus (L.) R.Br. & - & Smut grass & Year around & $\begin{array}{l}\text { Lowlands of dry or arid zones, usually } \\
\text { in open and disturbed zone }\end{array}$ & Weed \\
\hline 79 & Sporobolus virginicus (L.) Kunth & - & $\begin{array}{l}\text { Drop seeds/ Sacaton } \\
\text { grass }\end{array}$ & October-February & Wet lowlands & Weed \\
\hline 80 & Urochloa panicoides $\mathrm{P}$. Beauv. & - & $\begin{array}{l}\text { Kuri millet, Liverseed } \\
\text { grass }\end{array}$ & July-December & $\begin{array}{l}\text { Low grasslands, paddy fields, wet } \\
\text { sandy shores etc. }\end{array}$ & Weed, Cover grass (Skerman and Riveros, 1990) \\
\hline 81 & Zoysia matrella (L.) Merr. & - & $\begin{array}{l}\text { Korean/Manila temple } \\
\text { grass }\end{array}$ & March-September & Dry and wet zone and sandy soil & $\begin{array}{l}\text { Lawn grass, fodder, soil binder (Skerman and } \\
\text { Riveros, 1990) }\end{array}$ \\
\hline
\end{tabular}




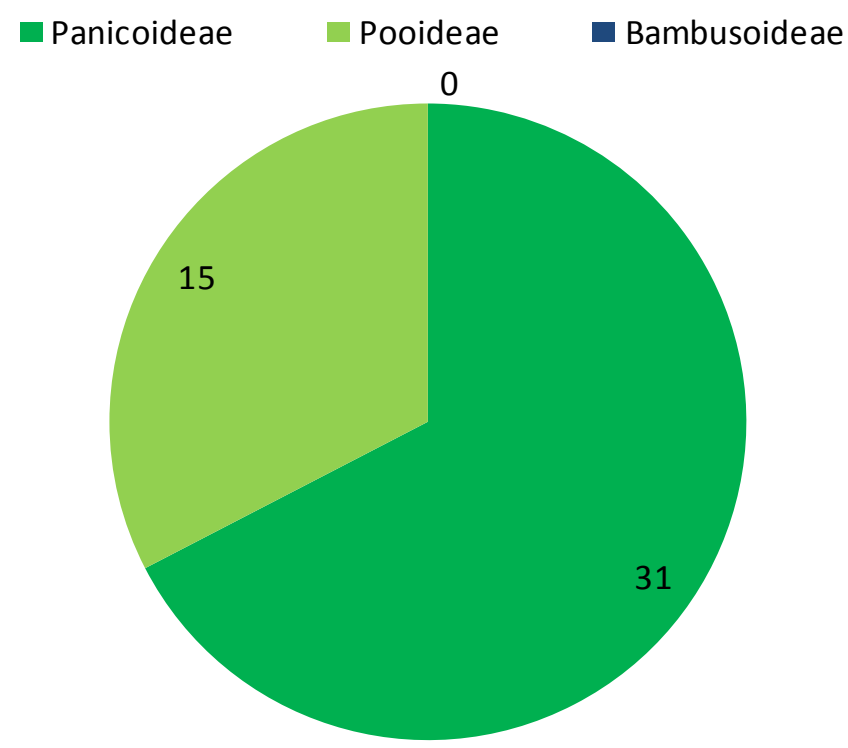

Fig. 1. Subfamilial distribution of genera of the family Poaceae from the BAU campus

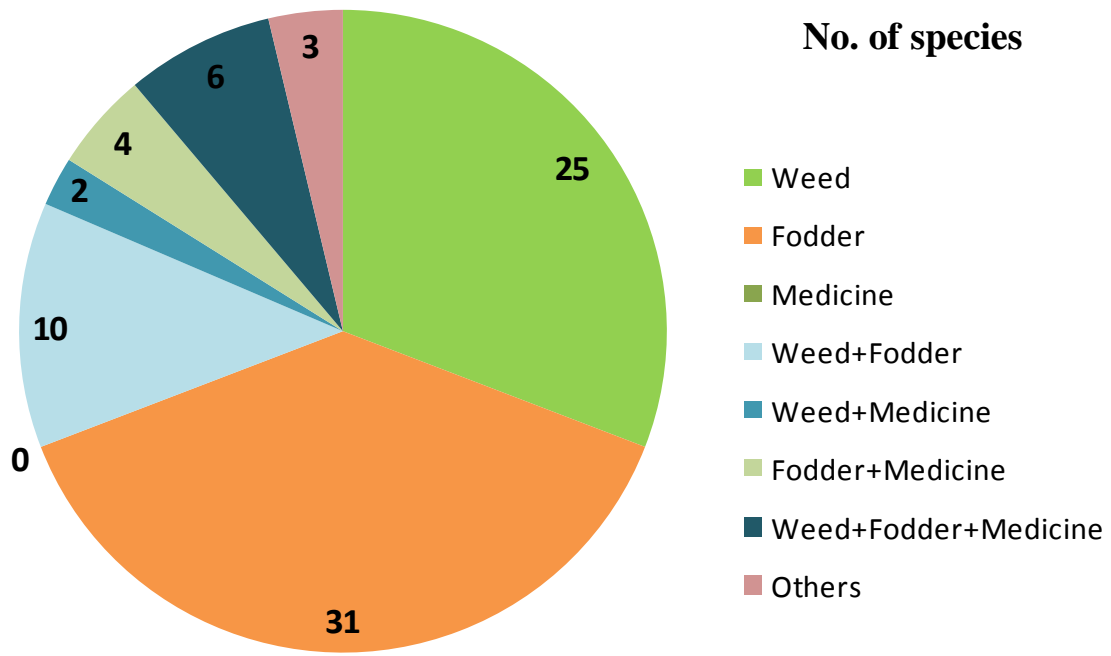

Fig. 2. Ethnobotanical uses of the species from the BAU campus

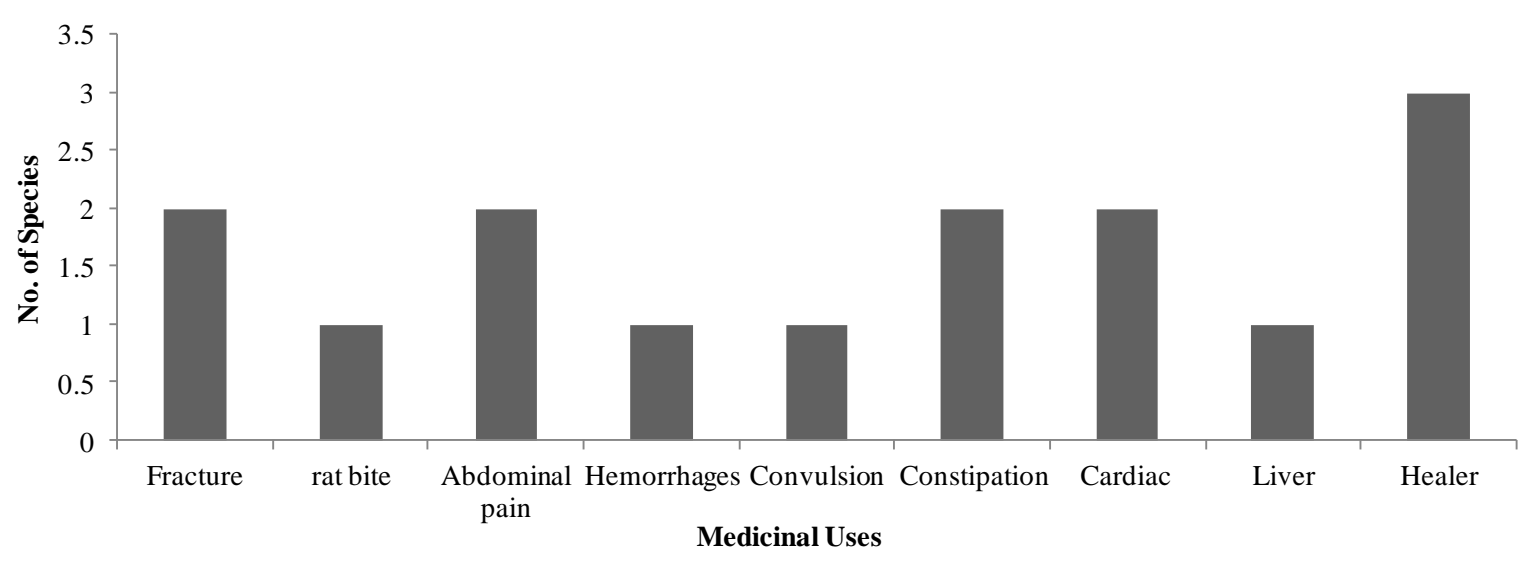

Fig. 3. Number of species from the BAU campus used for different ethnomedicinal purposes 


\section{Conclusion}

A sum of 81 species under 46 genera and 2 subfamilies (Panicoideae and Pooideae) of the family Poaceae were collected and documented their use in various ailments. Out of the three subfamilies, weeds from Bambusoideae were not found. Among the genera, Digitaria, Eragrostis, Brachiaria, Panicum, Echinochloa and Sporobolus were most dominant, while 28 genera were found with single species in BAU campus. Cynodon dactylon, Echinochloa crus-galli, E. colona, Eleusine indica and Imperata cylindrica are major and common obnoxious weeds for rice, wheat and other crop fields. The flowering period of these weeds will be helpful for the management of weed population. A good number of these weeds have various economic, ethnomedicinal and other uses. Many of them are good fodder, soil binder, used as lawn and turf grass and have high medicinal value. The knowledge generated from the present research would be helpful for the management practices of grass weeds as well as for getting high economic benefits from beneficial species.

\section{Acknowledgements}

We grateful to Dr. S. Nasir Uddin, Principal Scientific Officer, Bangladesh National Herbarium for identification of some of the specimens, and acknowledge the Ministry of Science and Technology, Government of the People's Republic of Bangladesh.

\section{References}

Ahmed, Z.U., Hassan, M.A., Begum, Z.N.T., Khondker, M., Kabir, S.M.H., Ahmad, M., Ahmed A.T.A., Rahman, A.K.A. and Hoque, E.U. (eds). 2008. Encyclopedia of Flora and Fauna of Bangladesh, Vol. 12. Angiosperms: Monocotyledons (Orchidaceae - Zingiberaceae). pp. 152-425. Asiatic Society of Bangladesh, Dhaka.

Bor, N.L. 1960. The Grasses of Burma, Ceylon, India and Pakistan. pp. 767. Pergamon Press, London.

Bouchenak-khelladi, Y., Verboom, G.A., Savolainen, V. and Hodkinson, T.R. 2010. Biogeography of the grasses (Poaceae): a phylogenetic approach to reveal evolutionary history in geographical space and geological time. Botanical Journal of the Linnean Society, 162: 543-557. https://doi.org/10.1111/j.1095-8339.2010.01041.x

Bryson, C.T. and Carter, R. 2008. The significance of Cyperaceae as weeds. Monograph Systematic Botany Missouri Botanical Garden, 108: 15-101.

Christenhusz, M.J.M. and Byng, J.W. 2016. The number of known plants species in the world and its annual increase. Phytotaxa 261: 201-217. https://doi.org/10.11646/ phytotaxa.261.3.1

Gilliland, H.B. 1971. Flora of Malaya - Grasses. pp. 1-319. The Botanic Gardens, Singapore

PMCid:PMC2139034

Holm, L.G., Plucknett, D.L., Pancho, J.V., Herberger, J.P. 1977. The World's Worst Weeds: Distribution and Biology. University Press of Hawaii, Honolulu.

Jannat-E-Tajkia, Sagar, A. and Sarwar, A.K.M. Golam. 2018. Reassessment of cyperaceous weed biodiversity at Bangladesh Agricultural University campus. Journal of Bangladesh Agricultural University, 16: 221-226. https://doi.org/10.3329/jbau.v16i2.37964

Kirtikar, K., Basu, B.D. and an, I.C.S. 1935. Indian Medicinal Plants, Vols. 1-4. Bishen Singh Mahendra Pal Singh, Dehra Dun, India.

Lym, R.G. and Travnicek, A.J. 2015. Identification and control of invasive and troublesome weeds in North Dakota. pp. 1-76. NDSU Extension Service, USA.

Mannetje, L.T. and Jones, R.M. (eds.) 1992. Plant Resources of SouthEast Asia (Forages). pp. 1-300. Pudoc Scientific Publishers, The Netherland.

Noor, K., Khan, E.A., Baloch, M.S., Khan, M.A., Awan, I.U., Sadiq, M., Aslam, M. 2012. Allelopathic effect of congress grass on weeds and yield of wheat. Pakistan Journal of Weed Science Research, 18: 307-318.

Pal, D.C. and Jain, S.K. 1998. Tribal Medicine. pp. 1-317. Naya Prakash, India.

Purseglove, J.W. 1968. Tropical Crops (Monocotyledons). pp. 1-607. Longman Gr. Ltd., U.K.

Sarwar, A.K.M. Golam and Prodhan, A.K.M.A. 2011. Study on the Cyperaceous weeds of Bangladesh Agricultural University campus. Journal of Agroforestry and Environment, 5: 8991.

Shabi, T.H., Islam, A.K.M.M, Hasan, A.K., Juraimi, A.S. and Anwar, M.P. 2018. Differential weed suppression ability in selected wheat varieties of Bangladesh. Acta Scientifica Malaysia 2: 1-7. https://doi.org/10.26480/asm.02.2018.01.07

Shear, G.M. 1985. Introduction and history of limited tillage. In: Wiese, A.F. (ed.), Weed Control in Limited-tillage Systems. pp. 1-14. Weed Science Society of America, USA.

Skerman, P.J. and Riveros, F. 1990. Tropical Grasses. pp. 1-832. FAO, Italy.

Tothill, J.C. and Backer, J.B. 1983. The grasses of southeast Queensland. pp. 1-300. University of Queensland Press, Queensland.

Uddin, S.N. 2006. Traditional uses of ethnomedicinal plants of the Chittagong Hill Tracts. pp. 1-992. Bangladesh National Herbarium, Dhaka. PMid:17010360

UNDP. 1988. Land resource appraisal of Bangladesh for agricultural development report 2: Agroecological Regions of Bangladesh. pp. 577. UNDP and FAO, Italy.

USDA. 2012. National Resource Conservation Service: Plant Database. Retrieved October 12, 2012, from http://plants.usda.gov/java/ 